\title{
I-123-MIBG cardiac innervation imaging in patients with atrial fibrillation
}

\author{
Anna Teresińska, $\mathrm{PhD}^{\mathrm{a}}$ \\ a The Cardinal Stefan Wyszyński Institute of Cardiology, Warsaw, Poland
}

Received Jan 6, 2019; accepted Jan 7, 2019

doi:10.1007/s12350-019-01601-6

\section{See related article, pp. 1939-1950}

Atrial fibrillation (AF) is a supraventricular tachyarrhythmia with uncoordinated atrial activation and consequently ineffective atrial contraction. ${ }^{1} \mathrm{AF}$ is one of the most important health problems in developed countries, with the prevalence of approximately $3 \%$ in adults aged 20 years or older, significantly increasing with advancing age. It is associated with an increased risk of stroke (five-fold), heart failure (three-fold), and dementia (two-fold). This arrhythmia is independently associated with nearly a two-fold increased risk of allcause mortality, especially cardiovascular mortality due to sudden death, heart failure, or stroke. ${ }^{1,2}$

AF occurs when structural and/or electrophysiological abnormalities alter atrial tissue to promote abnormal impulse formation and/or propagation. A focal source in the pulmonary veins (PVs) can trigger AF, and ablation of this source can suppress recurrent AF, what led to the development of pulmonary vein isolation (PVI) as the foundation for radiofrequency catheter ablation methods. Although the PVs are the most common sites for ectopic focal triggers, experimental and clinical studies have shown that the intrinsic cardiac autononomic nervous system (CANS) plays an important role in the initiation and maintenance of AF. The system primarily includes thousands of autonomic neurons and nerves located in ganglionated plexuses (GPs), which are located in the epicardial fat pads. There are 7 major GPs, including 4 located in the left atrium (LA)

Reprint requests: Anna Teresińska, PhD, The Cardinal Stefan Wyszyński Institute of Cardiology, Alpejska 42, 04-628 Warsaw, Poland; ateresinska@ikard.pl

J Nucl Cardiol 2020;27:1951-4.

$1071-3581 / \$ 34.00$

Copyright (c) 2019 American Society of Nuclear Cardiology. around the PVs. However, the usefulness of ablation of autonomic ganglia as an initial or repeat ablation strategy for paroxysmal, persistent, and long-standing persistent AF is not well established. The effectiveness of ablation of GPs in patients with AF remains controversial and one of the major reasons can be the lack of an efficient method to localize the GPs. The standard approach is to apply high-frequency stimulation (HFS) to the presumed GP areas to induce their typical vagal reaction (i.e., elicit AV block). As HFS has low specificity and sensitivity, is invasive and time consuming, better methods for localization of GPs are needed. ${ }^{1-3}$

The adrenergic part of CANS can be evaluated with I-123-metaiodobenzylguanidine (MIBG), which is a norepinephrine analog and a tracer for sympathetic neuron integrity and function. ${ }^{4}$ MIBG imaging has been performed to assess patients with AF, for: (1) the prognosis of patients with paroxysmal atrial fibrillation $(\mathrm{PAF})^{5,6}$; (2) the prediction of outcome of catheter ablation of $\mathrm{AF}^{7,8}$; and (3) evaluation of denervation/ renervation after AF ablation. ${ }^{8,9}$

In patients with PAF and without structural heart disease, CAS abnormality defined as reduced $\mathrm{H} / \mathrm{M}$ ratio from planar MIBG delayed imaging was a predictor of vascular events (myocardial infarction, stroke, or heart failure) during a mean of 4.5 years follow-up. Therefrom, MIBG imaging in this group of patients could support clinical risk stratification. ${ }^{5}$ In a group of patients with first occurrence of PAF, the same investigators identified a reduced $\mathrm{H} / \mathrm{M}$ ratio as a predictor for the development of permanent AF during a mean of 4 years follow-up. Hence, MIBG imaging may be a useful modality for predicting the development of AF. ${ }^{6}$ If the use of planar MIBG scintigraphy to risk stratify patients with PAF is wider validated, this widely available method can improve patient selection for AF ablation. The expensive and complicated ablation technique could potentially be avoided in patients who would derive little or no benefit. ${ }^{10}$

Arimoto et al. demonstrated that a high global washout rate of MIBG (calculated in a stable sinus 
rhythm condition 5 days after PVI) is an independent predictor of AF relapses during a mean follow-up period of 14 months in patients with either paroxysmal or permanent AF. These results indicate that excessive sympathetic nervous activation may be one of the mechanisms of AF recurrences, and the evaluation of the cardiac nerve activity using planar MIBG scintigraphy shortly after the AF ablation may be a promising tool to predict the patient's outcome. ${ }^{7}$ Wenning et al. performed planar and SPECT MIBG imaging in a small group of 16 PAF patients before and 4 weeks after PVI. For the short-term follow-up (6 months), the results suggested that the presence of regional innervation defects after PVI on MIBG SPECT images may be indicative for the risk of AF relapses. ${ }^{8}$

MIBG SPECT allows assessment of the impact of $\mathrm{AF}$ ablation on ventricular myocardial innervation. The study performed by Wenning et al. showed the deterioration of left ventricular (LV) MIBG uptake after PVI, observed in SPECT images performed 4 weeks after the ablation procedure, in 10 of $16(63 \%)$ PAF patients. ${ }^{8}$ In the study performed by Lemery et al. on 5 patients (4 with PAF and 1 with persistent $\mathrm{AF}$ ), radiofrequency ablation targeted LA GPs identified by HFS, in addition to PVI. The planar and SPECT MIBG imaging was performed before, early (1-8 days), and late (34 months) after ablation. Ventricular myocardial postablation denervation was documented in all patients and persisted at least 3-4 months post-ablation. ${ }^{9}$ For $\mathrm{AF}$ ablation procedures it had been shown that successful elimination of AF usually involves various degrees of denervation of the LA. Association of catheter ablation, which is technically performed in the LA area (PVs ostia, GPs), with modifications of autonomic tone in the $\mathrm{LV}$, is obvious: intrinsic CANS is a distributed network of ganglia and interconnecting nerves, and sympathetic postganglionic somata in each intrinsic cardiac GP project axons to widespread regions of the heart. ${ }^{11}$ The studies by Wenning et al. and by Lemery et al. proved for the early stage after AF ablation that by conventional planar and SPECT MIBG imaging 'the autonomic footprint left by atrial ablation could be visualized in the ventricle'. ${ }^{9}$ It was not explored by Wenning or by Lemery how long this effect is maintained over 4 months. Observing another serious intervention on the heart, LV adrenergic reinnervation appeared approximately 6 months after transmyocardial laser revascularisation (TMLR) in the assessment by MIBG SPECT. Although TMLR-induced impairment of CANS could contribute to the clinical improvement shortly after the procedure, the good clinical effect was lasting for years, what was linked to another involved processincreasing angiogenesis. ${ }^{12}$ In patients after heart transplantation (HTx), sympathetic reinnervation of cardiac allografts was in large part evaluated by norephinephrine analogs scintigraphy and it proved to be partial, heterogeneous, occurring in some but not all recipients (mostly at around 2 years, but in some patients as early as 5-6 months after HTx), never reaching global cardiac reinnervation. ${ }^{13}$ For AF ablation, in a randomized study it had been shown, that GP ablation can improve clinical outcomes (freedom from AF) up to 24 months after the sole procedure in $48 \%$ of patients and after the adjunct to PVI in $74 \% .{ }^{14}$ Effects of circumferential PVI on cardiac autonomic function were assessed by deceleration and acceleration capacity of heart rate and immediate decrease of autonomic function persisted after PVI for at least 1 year. ${ }^{15}$ The long-term ( $>2$ years) clinical outcomes and innervation results of GPs ablation have not been studied and whether reinnervation causes recurrent $\mathrm{AF}$ post-ablation remains uncertain. ${ }^{16}$ The dynamics of changes over time in LV innervation after AF ablation can be tracked with MIBG as it was shown by Wenning and Lemery ${ }^{8,9}$ and it could be related to AF relapses.

The papers dedicated to AF disease and MIBG, as selected above, technically were not based on the measurements performed in the atrial region solely. They included planar studies of the global cardiac sympathetic activity and SPECT applied as a tool of LV and not atria sympathetic activity assessment. Similarly, a new generation of cardiology dedicated cadmium zinc telluride (CZT) SPECT cameras have been applied in patients with AF for global and LV cardiac assessment. ${ }^{17}$ Solidstate technology offers significantly improved imaging parameters, including sensitivity and spatial resolution, allowing high quality SPECT imaging with spatial resolution approximately twice better than in SPECT based on classical Anger scintillation detector (about $5 \mathrm{~mm}$ vs $\geq 10 \mathrm{~mm}$ ). ${ }^{18}$ This suggests that CZT SPECT has the potential to identify the small structures, like GPs located on the atrial epicardium, which typically measure $5-10 \mathrm{~mm}$. The results of CZT SPECT studies of atria, particularly in GP diagnostics in patients with AF, have not been published but few congress abstracts (always with involvement of Royal Brompton Hospital, London, United Kingdom, like in the case of discussed study), and a case report written by the other group. ${ }^{19}$

In this issue of the Journal, Stirrup et al. have addressed the usefulness of cardiac MIBG scintigraphy for non-invasive identification of LA GPs. ${ }^{20}$ It would be of clinical importance for the ablation of the plexi, as a potential add-on to PVI in patients with PAF, if MIBG SPECT could replace invasive HFS technique with its well recognized limitations for GPS identification. The idea behind the presented method is that localization of GPs by invasive multi-site testing HFS, a process that identifies GPs by their typical parasympathetic response 
of slowing atrio-ventricular nodal conduction, and noninvasive imaging of LA sympathetic innervation by MIBG, might identify the same areas, as sympathetic and parasympathetic fibers co-localize in GPs. The work of Stirrup et al. is a complex, all-encompassing approach to introduction of a relatively young technology (CZT SPECT-CT) to the new clinical applications (LA GPs ablation). Phantom studies of specific design preceded clinical studies to demonstrate feasibility of the technique and to establish the methods for image acquisition and interpretation. Thereafter, in the pilot group of 20 patients with $\mathrm{AF}$, the validating assessment of the technique, including inter- and intra-observer variability and inter-study variability, was carried on. Finally, correlation with HFS was performed. The study is elegant and the achieved results are encouraging. The proposed diagnostic approach is built on demanding hardware and software supporting the CZT SPECT ('DSPECT') imaging. First, high-resolution reconstruction was necessary for imaging GPs: a very good D-SPECT resolution $(5.6 \mathrm{~mm})$ yielded by standard iterative reconstruction, may be a borderline resolution for identification of subcentimeter size GPs $(0.5-1 \mathrm{~cm})$. Therefore, the high-resolution reconstruction algorithm was developed and validated on the NEMA resolution phantom for MIBG data, leading up to the resolution of $3.9 \mathrm{~mm}$. Second, simultaneous cardiorespiratory gating using a dedicated device was needed as well as software upgrade to allow processing minimizing the effects of motion on spatial localization of MIBG uptake. Third, the necessary images and calculations were generated by a dedicated workstation (SUMO D-SPECT). SUMO optional application enables assessment of the sympathetic innervation of the heart by quantification of uptake ratios between regions of interest, identifying discreet uptake areas (DUAs) of MIBG. In SUMO, the nuclear data from D-SPECT can be effectively coupled with CT scans and with physiological signals. Following registration, the semi-automatically CT-derived LA segment is used as an anatomical constraint to define a region of search (ROS) around the LA endocardium to facilitate identification of focal MIBG uptake adjacent to the atria. Focal increased MIBG activity within the ROS is automatically overlaid on the CT-derived LA surface, generating a hybrid 3D image of LA innervation and anatomy. The results of the study showed, that MIBG solid-state SPECT LA innervation imaging identifies GPs verified by HFS with good accuracy and reproducibility, particularly when reconstructed with cardiorespiratory gating. However, DUAs identified over the lateral and inferior LA walls were less likely to be HFS positive, probably due to difficulty in distinguishing true LA epicardial activity from activities in neighboring structures. ${ }^{20}$
The usefulness of ablation of autonomic ganglia as an initial or repeat ablation strategy for AF is not well established, as the results of randomized studies are not unequivocal. Further studies are needed to better understand the role of GPs ablation for rhythm control. As timing and extent of reinnervation is dependent on the extent of denervation, and as reinnervation is not an isolated effect of the denervating intervention on the heart, long-term clinical benefits of GPs ablation are to be investigated. Regardless of a clinical settlement to the on-going dispute, the study by Stirrup et al. has shown the possibilities of CZT SPECT-CT using I-123-MIBG for a truly SPECT LA innervation imaging. The preliminary results are encouraging but need further validation in larger trials. It is possible, that the presented methodology will not replace invasive HFS in identification of GPs, as the predictive value of receiving HFS-positive response at DUA area was only $76 \%$, mainly because of DUAs located either laterally/inferiorly on LA MIBG images or identified with less confidency. On the other hand, the criteria that define a positive response to HFS are still debated and MIBG results may help in better selection of LA regions to be submitted to ablation, beyond HFS results. Moreover, MIBG-CZT-SPECT-CT technique poses an innovative tool for the assessment of the extent of LA denervation and the dynamics of renervation, at least in some regions, after ablation. It should be stressed that the presented methodology is developed for a specific scintigraphic system with a dedicated application software for the fusion of D-SPECT scintigraphic images with CT scans and with electrophysiological signals, and for the identification of discreet uptake areas of MIBG. ${ }^{20}$ The natural alternative for MIBG-CZT-SPECT-CT technique could be PET-CT. Taking into account its high sensitivity, spatial resolution, existing advanced software for reconstruction and fusion, proved ability to image small uptake areas within heart (for example, for identification of high-risk atherosclerotic plaques in coronary $\operatorname{arteries}^{21}$ ), and advanced development of new F-18 labeled tracers of cardiac innervation, ${ }^{22}$ PET-CT has a high potential to apply to atrial diagnostics.

\section{Disclosure}

The author has no conflict of interest to disclose.

\section{References}

1. January CT, Wann LS, Alpert JS, Calkins H, Cigarroa JE, Cleveland JC Jr, et al. 2014 AHA/ACC/HRS guideline for the management of patients with atrial fibrillation: a report of the American College of Cardiology/American Heart Association 
Task Force on Practice Guidelines and the Heart Rhythm Society. J Am Coll Cardiol. 2014;64:e1-76.

2. Kirchhof P, Benussi S, Kotecha D, Ahlsson A, Atar D, Casadei B, et al. 2016 ESC Guidelines for the management of atrial fibrillation developed in collaboration with EACTS. Eur Heart J. 2016;37:2893-962.

3. Calkins H, Hindricks G, Cappato R, Kim Y-H, Saad EB, Aguinaga L, et al. 2017 HRS/EHRA/ECAS/APHRS/SOLAECE expert consensus statement on catheter and surgical ablation of atrial fibrillation. Europace. 2017;20:e1-160.

4. Chirumamilla A, Travin MI. Cardiac applications of 123I-mIBG imaging. Semin Nucl Med. 2011;41:374-87.

5. Akutsu Y, Kaneko K, Kodama Y, Li HL, Suyama J, Shinozuka A, et al. Significance of cardiac sympathetic nervous system abnormality for predicting vascular events in patients with idiopathic paroxysmal atrial fibrillation. Eur J Nucl Med Mol Imaging. 2010;37:742-9.

6. Akutsu Y, Kaneko K, Kodama Y, Li H-L, Suyama J, Shinozuka A, et al. Iodine- $123 \mathrm{mIBG}$ imaging for predicting the development of atrial fibrillation. JACC Cardiovasc Imaging. 2011;4:78-86.

7. Arimoto T, Tada H, Igarashi M, Sekiguchi Y, Sato A, Koyama T, et al. High washout rate of iodine-123-metaiodobenzylguanidine imaging predicts the outcome of catheter ablation of atrial fibrillation. J Cardiovasc Electrophysiol. 2011;22:1297-304.

8. Wenning C, Lange PS, Schulke Ch, Vrachimis A, Monnig G, Schober O, et al. Pulmonary vein isolation in patients with paroxysmal atrial fibrillation is associated with regional cardiac sympathetic denervation. EJNMMI Res. 2013;3:81.

9. Lemery R, Ben-Haim S, Wells G, Ruddy T. I-123-Metaiodobenzylguanidine imaging in patients with atrial fibrillation undergoing cardiac mapping and ablation of autonomic ganglia. Heart Rhythm. 2017;14:128-32.

10. Ovadia M, Dugue KS. The Potential Utility of 123I-mIBG in Atrial Fibrillation and in the Electrophysiology Laboratory. Curr Cardiol Rep. 2012;4:200-7.

11. Armour JA. Potential clinical relevance of the "little brain" on the mammalian heart. Exp Physiol. 2008;93:165-76.

12. Teresinska A, Sliwinski M, Konieczna S, Szumilak B, Szymanska $\mathrm{M}$, Debski A, et al. Changes in cardiac adrenergic nervous system after transmyocardial laser revascularisation assessed by I-123MIBG SPECT. A preliminary report. Kardiol Pol. 2004;60:15-26.

13. Awad M, Czer LSC, Hou M, Golshani SS, Goltche M, De Robertis $\mathrm{M}$, et al. Early denervation and later reinnervation of the heart following cardiac transplantation: a review. J Am Heart Assoc. 2016;5:e004070.

14. Katritsis DG, Pokushalov E, Romanov A, Giazitzoglou E, Siontis GCM, Po SS, et al. Autonomic denervation added to pulmonary vein isolation for paroxysmal atrial fibrillation: a randomized clinical trial. J Am Coll Cardiol. 2013;62:2318-25.

15. Bauer A, Deisenhofer I, Schneider R, Zrenner B, Barthel P, Karch $\mathrm{M}$, et al. Effects of circumferential or segmental pulmonary vein ablation for paroxysmal atrial fibrillation on cardiac autonomic function. Heart Rhythm. 2006;3:1428-35.

16. Stavrakis S, Po S. Ganglionated Plexi Ablation: physiology and Clinical Applications. Arrhythm Electrophysiol Rev. 2017;6:18690.

17. Bellevre D, Manrique A, Legallois D, Bross S, Baavour R, Roth N, et al. First determination of the heart-to-mediastinum ratio using cardiac dual isotope (123I-MIBG/99mTc-tetrofosmin) CZT imaging in patients with heart failure: the ADRECARD study. Eur J Nucl Med Mol Imaging. 2015;42:1912-9.

18. Gambhir SS, Berman DS, Ziffer J, Nagler M, Sandler M, Patton J, et al. A novel high-sensitivity rapid-acquisition single-photon cardiac imaging camera. J Nucl Med. 2009;50:635-43.

19. Romanov A, Minin S, Breault Ch, Pokushalov E. Visualization and ablation of the autonomic nervous system corresponding to ganglionated plexi guided by D-SPECT 123ImIBG imaging in patient with paroxysmal atrial fibrillation. Clin Res Cardiol. 2017;106:76-8

20. Stirrup J, Gregg S, Baavour R, Roth N, Breault Ch, Agostini D, et al. (2019) Hybrid solid-state SPECT/CT left atrial innervation imaging for identification of left atrial ganglionated plexi: technique and validation in patients with atrial fibrillation. $\mathrm{J}$ Nucl Cardiol.

21. Joshi NV, Vesey AT, Williams MC, Shah AS, Calvert PA, Craighead FH, et al. 18F-fluoride positron emission tomography for identification of ruptured and high-risk coronary atherosclerotic plaques: a prospective clinical trial. Lancet. 2014;383:70513.

22. Werner RA, Chen X, Hirano M, Rowe SP, Lapa C, Javadi, Higuchi T. SPECT vs. PET in cardiac innervation imaging: clash of the titans. Clin Transl Imaging. 2018;6:293-303.

Publisher's Note Springer Nature remains neutral with regard to jurisdictional claims in published maps and institutional affiliations. 